\title{
ARTICLE \\ Desmoglein 2, but not desmocollin 2, protects intestinal epithelia from injury
}

\author{
Annika Gross ${ }^{1}$, Lotta A. P. Pack ${ }^{1}$, Gabriel M. Schacht ${ }^{1}$, Sebastian Kant ${ }^{2}$, Hanna Ungewiss ${ }^{3}$, Michael Meir ${ }^{4}$, Nicolas Schlegel ${ }^{4}$, \\ Christian Preisinger ${ }^{5}$, Peter Boor ${ }^{6}$, Nurdan Guldiken ${ }^{1}$, Claudia A. Krusche ${ }^{2}$, Gernot Sellge ${ }^{1}$, Christian Trautwein ${ }^{1}$, Jens Waschke ${ }^{4}$, \\ Arnd Heuser ${ }^{7}$, Rudolf E. Leube ${ }^{2}$ and Pavel Strnad ${ }^{1}$
}

Desmosomes are the least understood intercellular junctions in the intestinal epithelia and provide cell-cell adhesion via the cadherins desmoglein (Dsg)2 and desmocollin (Dsc)2. We studied these cadherins in Crohn's disease (CD) patients and in newly generated conditional villin-Cre DSG2 and DSC2 knockout mice (DSG2 ${ }^{\triangle I E C}$; DSC2 ${ }^{\triangle I E C}$ ). CD patients exhibited altered desmosomes and reduced Dsg2/Dsc2 levels. The intestines of both transgenic animal lines were histopathologically inconspicuous. However, DSG2 ${ }^{\triangle I E C}$, but not DSC2 ${ }^{\triangle I E C}$ mice displayed an increased intestinal permeability, a wider desmosomal space as well as alterations in desmosomal and tight junction components. After dextran sodium sulfate (DSS) treatment and Citrobacter rodentium exposure, DSG2 ${ }^{\triangle I E C}$ mice developed a more-pronounced colitis, an enhanced intestinal epithelial barrier disruption, leading to a stronger inflammation and activation of epithelial pSTAT3 signaling. No susceptibility to DSS-induced intestinal injury was noted in DSC2 ${ }^{\Delta I E C}$ animals. Dsg2 interacted with the cytoprotective chaperone Hsp70. Accordingly, DSG2 ${ }^{\Delta \mathrm{IEC}}$ mice had lower Hsp70 levels in the plasma membrane compartment, whereas $D S C 2^{\triangle I E C}$ mice displayed a compensatory recruitment of galectin 3, a junction-tightening protein. Our results demonstrate that Dsg2, but not Dsc2 is required for the integrity of the intestinal epithelial barrier in vivo.

Mucosal Immunology (2018) 11:1630-1639; https://doi.org/10.1038/s41385-018-0062-z

\section{INTRODUCTION}

Epithelial cell layers in general provide an efficient barrier against the hostile environment. The intestinal epithelium has to facilitate both, protection of the underlying tissue from invading microorganisms and efficient uptake of nutrients and solutes. Intercellular junctions constitute a crucial part of this barrier and consist of tight junctions (TJs), adherens junctions (AJs), and desmosomes that together form the apical junctional complex (AJC). ${ }^{1} \mathrm{TJ}$ are the primary structures sealing the intercellular space. However, pro-inflammatory signaling in epithelial cells alters the TJ composition leading to reduced barrier function, thereby contributing to the pathogenesis of multiple prevalent intestinal disorders such as inflammatory bowel disease, celiac disease, or infectious gastroenteritis. ${ }^{2,3}$ This process is driven by molecular remodeling that results in decreased levels of claudin 1 and occludin and increased amounts of claudins 2 and $15 .{ }^{1,4}$ AJs are established mediators of cell-cell adhesion and play an important role in cell polarization and differentiation via the associated Wnt/ $\beta$-catenin pathway. ${ }^{1}$

Desmosomes represent the least-studied AJC component. They are, similar to AJs, important for cell adhesion and cytoskeletal anchorage. ${ }^{5}$ Their importance becomes evident in inherited cardiomyopathies that are caused by mutation in desmosomal genes and blistering skin disorders elicited by desmosomal autoantibodies. Desmosomes are also altered in microbial infections in that their constituents are cleaved by bacterial toxins and become targeted in adenoviral respiratory infections. ${ }^{6-8}$ Desmosomes bear structural similarities with hemidesmosomes that with the help of integrins connect the cells to the extracellular matrix. The crucial importance of hemidesmosomes was recently demonstrated in mice with intestinal epithelial cell-specific a6 integrin ablation that develop colitis with spontaneous progression into high-grade intestinal carcinoma. ${ }^{9}$

To connect the cells, desmosomes comprise transmembrane proteins termed desmosomal cadherins. These are subdivided into the desmoglein and desmocollin type (gene names: DSGs/ DSCs, protein names: Dsgs/Dscs). Although homo- and heterophilic interactions between the extracellular Dsg and Dsc domains have been described, the latter are preferred and accordingly, both protein types are needed for cellular adhesion. ${ }^{5,10}$ Dsgs/Dscs are expressed in a cell type-specific pattern with Dsc2 and Dsg2 being the most widespread family members and the major desmosomal cadherins of digestive epithelia. ${ }^{6}$ On the cytoplasmic side, Dsg2/Dsc2 are connected to the armadillo proteins plakoglobin (PG) and plakophilin 2 (Pkp2), which, together with desmoplakin (Dsp), facilitate the attachment to the keratin cytoskeleton. 5

Despite their presumed biological importance, only in vitro studies addressed the role of desmosomal cadherins in the intestine so far. The data available up to date suggest that

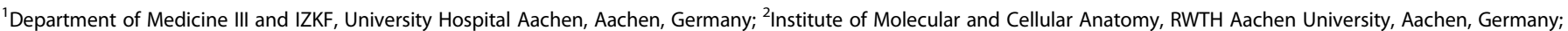

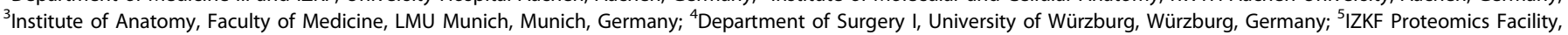

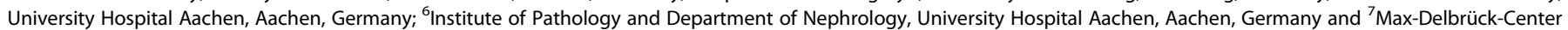
of Molecular Medicine, Berlin, Germany

Correspondence: Pavel Strnad (pstrnad@ukaachen.de)
}

Received: 16 February 2018 Revised: 14 June 2018 Accepted: 26 June 2018

Published online: 16 August 2018 
a
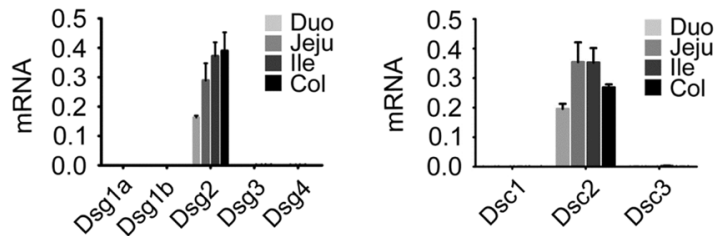

b
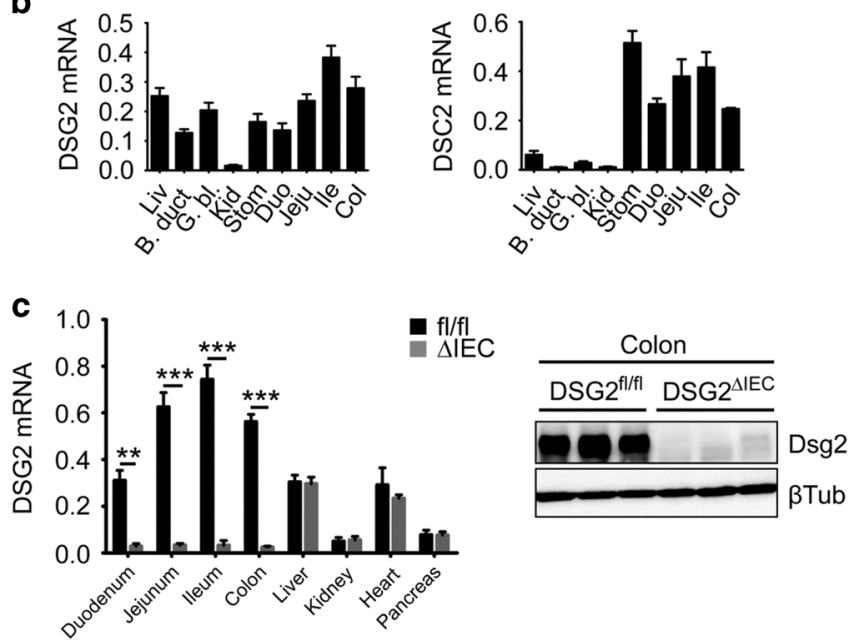

Fig. 1 Dsg2 and Dsc2 are the major cadherins expressed in the intestine and the generated DSG2-deficient animals (DSG2 ${ }^{\mathrm{IEC}}$ ) display an intestine-specific Dsg2 loss. a, b Expression levels of DSG2, DSC2 as well as the other desmoglein and desmocollin family members were assessed by real time RT-PCR in the indicated mouse organs $(n=4-5)$. c DSG2 mRNA expression was quantified in the highlighted mouse organs of DSG2 ${ }^{\Delta I E C}(\Delta \mathrm{IEC})$ and DSG2 ${ }^{\mathrm{f} / \mathrm{fl}}(\mathrm{fl} / \mathrm{fl})$ mice $(n=4)$ by real time RT-PCR. Colonic desmoglein 2 (Dsg2) protein levels were assessed in both groups by immunoblotting $(n=5)$. L7 (mouse ribosomal protein) gene and $\beta$-tubulin ( $\beta T u b)$ were used as an internal and a loading control, respectively. Dsg, desmoglein; Dsc, desmocollin; Liv, liver; B. duct, common bile duct; G.bl, gallbladder; Kid, kidney; Stom, stomach; Duo, duodenum; Jeju, jejunum; Ile, ileum; Col, colon. Two-tailed Student's $t$ test was used for statistical analyses. ${ }^{* *} p<0.01,{ }^{* * *} p<0.001$

although they are both functionally and structurally similar, they also display unique properties., ${ }^{6,11-13}$ For example, DSG2 knockdown resulted in a compensatory upregulation of $\mathrm{Dsc} 2$, whereas DSC2 loss did not lead to alterations in Dsg2 protein levels. ${ }^{14}$ Moreover, Dsg2 but not Dsc2 was cleaved during intestinal epithelial cell apoptosis and DSG2 downregulation inhibited this cell death pathway. ${ }^{15}$ In addition to experimental data pointing toward a functional importance of desmosomes, a selective decrease of Dsg2 was reported in patients with Crohn's disease. ${ }^{16}$ In cultured epithelial cells, this downregulation was associated with increased permeability that was prevented by stabilization of desmosomal adhesion. ${ }^{16}$ These disease-related changes coupled with their established in vitro biological function as well as the crucial importance of intercellular junctions ${ }^{1,9}$ prompted us to analyze the in vivo properties of desmosomal cadherins in the intestine. To that end, we generated conditional villin-Cremediated DSG2 knockout and DSC2 knockout (DSG2 ${ }^{\triangle \mathrm{IEC}} / \mathrm{DSC} 2^{\mathrm{DIEC}}$ ) mice and analyzed desmosomal alterations in Crohn's disease patients.

In summary, we demonstrate that intestinal DSG2 but not DSC2 is required for the integrity of the intestinal epithelial barrier in vivo. These data might be of human relevance, as Crohn's disease patients display altered desmosomes as well as reduced desmosomal cadherin levels.

\section{RESULTS}

As patients with Crohn's disease (CD) were reported to have reduced intestinal Dsg2 levels, ${ }^{16}$ we performed transmission electron microscopy that revealed an altered ultrastructure of the apical junctional complex (Suppl. Fig. 1A). In particular, TJs lacked proper membrane annealing, whereas desmosomes were sometimes missing and/or exhibited irregular or asymmetric plaques with reduced intermediate filament insertion. In contrast, AJ were largely unaltered (Suppl. Fig. 1A). In line with that and previous findings, ${ }^{16}$ a significant reduction in both desmosomal cadherins, i.e., Dsg2 and Dsc2, was seen, whereas no obvious alterations were detected in E-cadherin (Suppl. Fig. 1B, C).

Next, we directly addressed the biological relevance of desmosomal cadherins in the newly generated intestinal-specific knockouts. In agreement with previous reports, ${ }^{6}$ Dsg2 and Dsc2 were the only desmosomal cadherins expressed in the mouse intestine (Fig. 1a). Both cadherins were abundantly expressed in the intestine, whereas lower amounts were seen in other organs such as the kidney (Fig. 1b). The newly generated intestinespecific Dsg2 knockout (DSG2 ${ }^{\triangle \mathrm{IEC}}$ ) displayed a selective and efficient deletion of Dsg2 in both small and large intestine (Fig. 1c, Suppl. Fig. 2A-D) and no upregulation of other desmoglein and desmocollin isoforms was observed (Suppl. Fig. 3A). Immunofluorescence staining confirmed the loss of Dsg2, whereas Dsc2/ PG staining was retained and demonstrated an unaltered desmosome distribution (Fig. 2a). DSG2 ${ }^{\triangle I E C}$ mice developed normally, displayed normal weight gain and colon length (Suppl. Fig. 4). No diarrhea was observed. Histological examination revealed a morphologically inconspicuous small and large intestine with correctly differentiated and localized cell types (Fig. 2b, Suppl. Fig. 5A, B and data not shown). Unaltered levels of the stem cell markers LGR5 and R-spondin and no changes in the basal cell proliferation were noted (Suppl. Fig. 5C, D Suppl. Fig. 6). No inflammation was observed at any analyzed time point (Suppl. Fig. 7 and not shown). Electron microscopy revealed largely normal desmosomal plaques, whereas the intercellular space between the junctions was significantly wider in the small and large intestine (Fig. 2c, Suppl. Fig. 8). Biochemical analysis illustrated profound changes in other desmosomal components with upregulation of Dsc2 and downregulation of Dsp and PG, whereas the levels of Pkp2 and keratin 8 (K8) were not altered (Fig. 2d, Suppl. Fig. 9). The same alterations were observed in small and large intestine and the changes likely occurred at the posttranscriptional level, as the mRNA expression of all analyzed desmosomal genes was unaltered (Fig. 2e and data not shown). Immunoblotting revealed decreased levels of occludin and claudin 1 that are often reduced in situations leading to barrier dysfunction, whereas no differences were observed in the TJ proteins claudin 7 and 15 and the AJ protein E-cadherin (Suppl. Fig. 10A). Immunofluorescence staining revealed a normal distribution of tight and AJ proteins (Suppl. Fig. 10B). Myosin II regulatory light chain (MLC) phosphorylation, that constitutes a key regulator of TJ permeability, did not differ between DSG2 $2^{\triangle I E C}$ and $D S G 2^{\mathrm{f} / \mathrm{fl}}$ control mice (Suppl. Fig. 10C). In line with the described changes in epithelial junctions, DSG2 $2^{\triangle I E C}$ mice had an increased intestinal permeability (Fig. 2f).

Given that DSG2 ${ }^{\triangle I E C}$ mice did not show a spontaneous intestinal injury, we tested their susceptibility to dextran sodium sulfate (DSS) colitis. Even at low DSS doses, DSG2 ${ }^{\triangle \mathrm{IEC}}$ animals developed a profound weight loss with bloody diarrhea and intestinal lesions (Fig. $3 a-c)$. As an additional sign of tissue destruction, DSS-treated DSG2 ${ }^{\triangle I E C}$ mice had shorter colons (Fig. 3c). The histological examination revealed a marked epithelial loss with an edema, inflammatory reaction, and goblet 
a

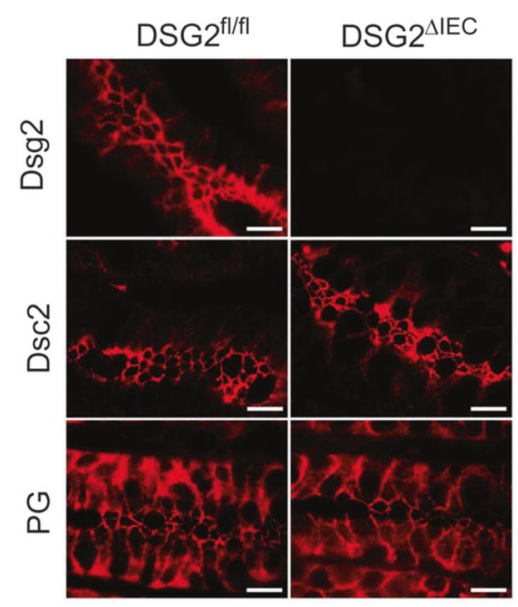

b

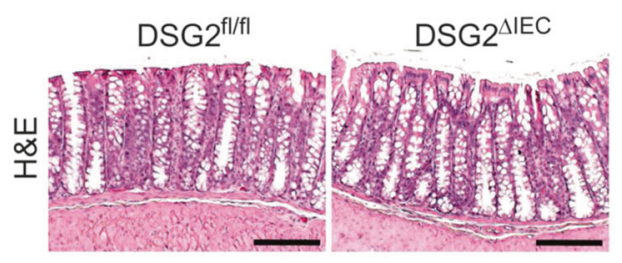

d

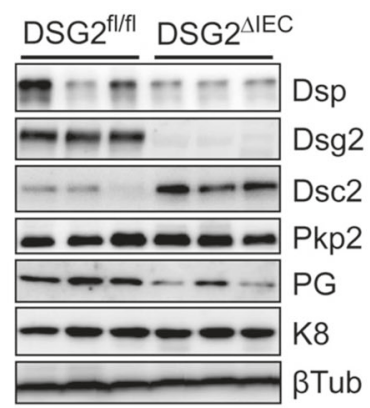

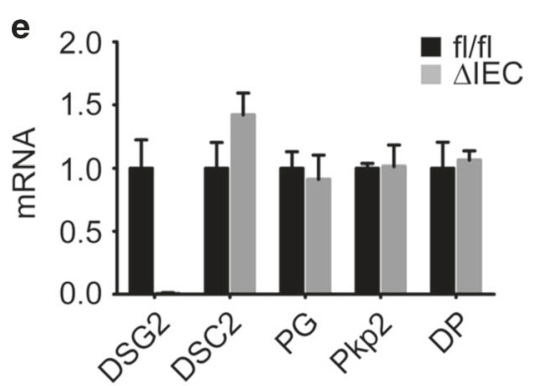

C

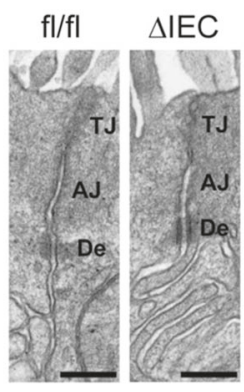

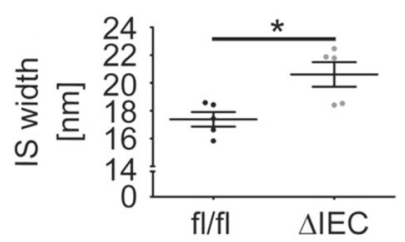

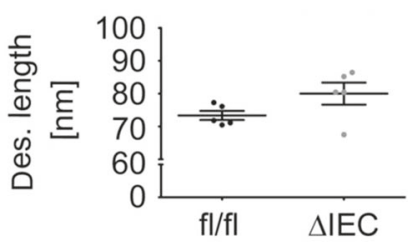

f

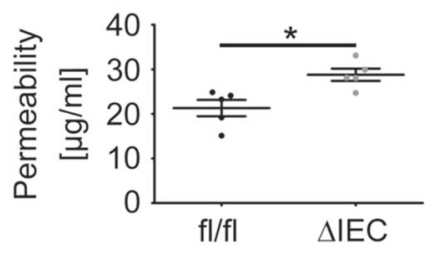

Fig. 2 DSG2-deficient animals $\left(D S G 2^{\Delta I E C}\right.$ ) display an altered desmosomal protein composition, a wider desmosomal space width and increased intestinal permeability. a The distribution of Dsc2, plakoglobin (PG) and Dsg2 in the colon of DSG2 $2^{\Delta \mathrm{IEC}}(\Delta \mathrm{IEC})$ and DSG2 $2^{\mathrm{fl} / \mathrm{fl}}(\mathrm{fl} / \mathrm{fl}) \mathrm{mice}$ was visualized by immunofluorescence. Scale bar $=20 \mu \mathrm{m}$. b Hematoxylin and eosin (H\&E) staining revealed an unperturbed overall colon architecture. c Desmosomal ultrastructure of colon samples was assessed by electron microscopy with subsequent quantification of the desmosomal intercellular space (IS) width and length of the desmosomal plaque $(n=5)$. Scale bar $=200 \mathrm{~nm}$. AJ, adherens junctions; De, desmosomes; TJ, tight junctions d, e Immunoblotting and RT-PCR were employed to study the impact of Dsg2 loss on desmosomal composition in the colon $(n=5)$. Dsc2, desmocollin 2; Dsp, desmoplakin; Pkp2, plakophilin 2; K8, keratin 8. f Administration of 4kD FITCdextran with subsequent quantification of serum FITC levels was utilized as a measurement of intestinal permeability $(n=5)$. Average mRNA expression in DSG2 ${ }^{\mathrm{f} / \mathrm{fl}}$ mice was arbitrarily set as 1 and levels in DSG2 ${ }^{\Delta \mathrm{IEC}}$ mice are presented as ratio. L7 (mouse ribosomal protein) gene and $\beta$-tubulin ( $\beta$ Tub) were used as an internal and a loading control, respectively. Data in c, $\mathbf{f}$ are shown as dot plots. Two-tailed Student's $t$ test was used for statistical analyses. ${ }^{*} p<0.05$

cell loss that resulted in significantly higher injury scores (Fig. 3d). The pronounced inflammation was confirmed by increased intestinal myeloperoxidase activity as well as by higher proinflammatory cytokine levels (Fig. 3e, f). Analysis of mesenteric lymph nodes demonstrated increased bacterial translocation (Fig. 3e).

To further delineate the mechanisms underlying the observed phenotype, we analyzed the effects of short-term DSS administration that led to modest histological changes (Fig. 4a). Compared with controls, DSG2 ${ }^{\mathrm{IEC}}$ animals displayed a more- profound epithelial cell loss into the intestinal lumen as demonstrated by higher levels of the epithelial marker K8 in the luminal content of the colon and by histological observation (Fig. 4a). In DSG2 ${ }^{\triangle \mathrm{IEC}}$ mice, DSS treatment resulted in a marked increase in intestinal permeability, whereas only a moderate DSS effect was seen in DSG2 ${ }^{\mathrm{f} / \mathrm{fl}}$ mice (Figs. 2f, 4b). In line with the more-pronounced epithelial leakiness, molecular analyses demonstrated an increased mRNA expression of pro-inflammatory cytokines and an activated IL-22-STAT3 signaling (Fig. 4c-e). Immunostaining revealed that STAT3 was phosphorylated in the 


\section{a}

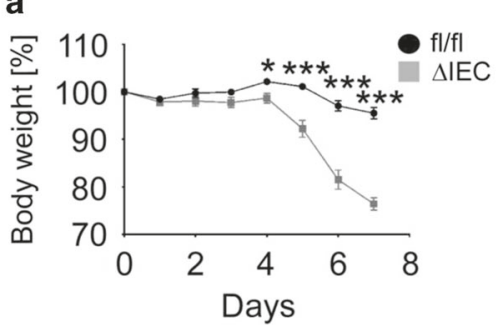

C
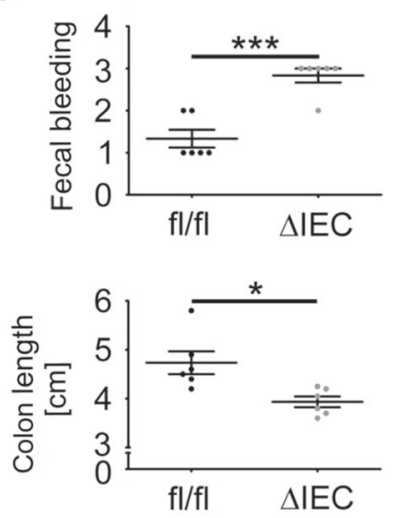

e
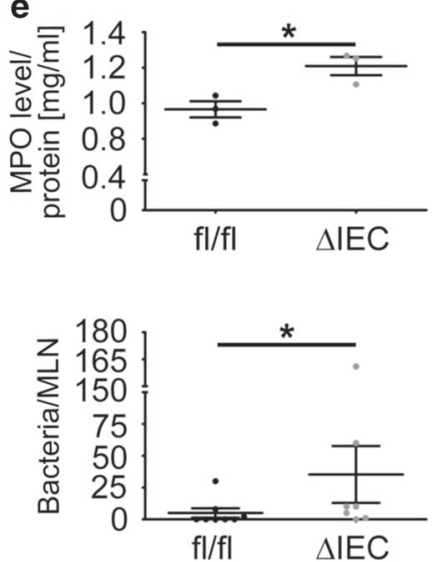

b
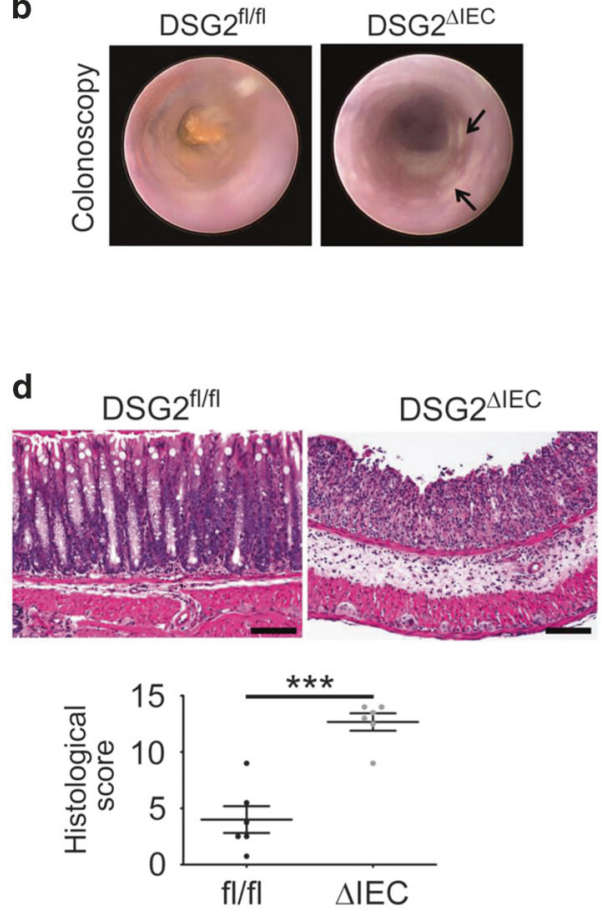

f
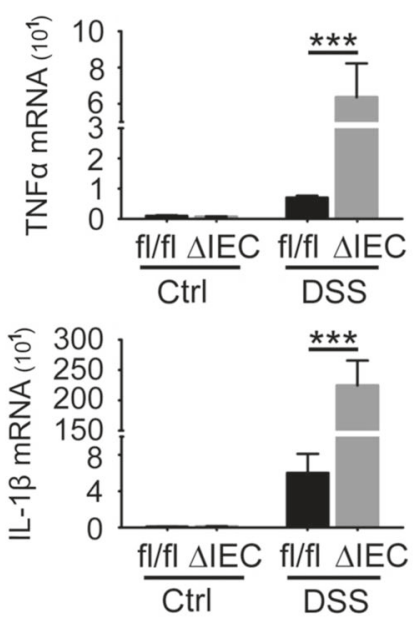

Fig. 3 DSG2-deficient animals (DSG2 ${ }^{\Delta \mathrm{IEC}}$ ) exhibit an enhanced susceptibility to dextran sodium sulfate (DSS)-induced colitis. a Relative body weights of DSG2 ${ }^{\Delta \mathrm{IEC}}(\triangle \mathrm{IEC})$ mice (gray rectangles) and their floxed littermates (black circles, DSG2 $2^{\mathrm{fl} / \mathrm{f}}, \mathrm{fl} / \mathrm{fl}, n=7$ each) were determined daily starting at the time of first DSS administration. b-d Macroscopic images of the colon mucosa obtained by colonoscopy, stool guaiac test with semi-quantitative scoring $(n=6)$, colonic length, and hematoxylin and eosin (H\&E) staining of colon sections with morphometric quantification were used to assess the severity of colitis at day 7 after DSS administration $(n=6)$. e, $\mathbf{f}$ The extent of colonic inflammation 7 days after DSS exposure was evaluated by myeloperoxidase (MPO) activity and real time RT-PCR for TNF $\alpha$ and IL-1 $\beta(n=3-6)$. The low cytokine expression in non-treated animals (ctrl) was arbitrarily set as 1. L7 (mouse ribosomal protein) gene was used as an internal control. Bacterial translocation into mesenteric lymph nodes (MLN) was quantified as the amount of colonies grown from total MLN homogenates on blood agar plates $(n=7-8)$. Data in $\mathbf{c}$, d, e are presented as dot plots. Two-tailed Student's t test was used for statistical analyses of DSS-treated animals. ${ }^{*} p<0.05,{ }^{* * *} p<0.001$

epithelial cells and is most likely responsible for the increased production of the antimicrobial peptide Reglll $\beta$ (Fig. 4d, f). As another consequence of the increased intestinal leakiness, we detected elevated levels of cleaved caspase 1 in DSS-treated $D S G 2^{\triangle I E C}$ mice suggesting enhanced inflammasome activation (Fig. 4e).

To test the susceptibility of DSG2 $2^{\triangle I E C}$ animals to infectious colitis, we exposed mice to the murine pathogen Citrobacter rodentium. In line with previous reports, the treatment did not lead to weight loss (Suppl. Fig. 11A). ${ }^{17}$ During the peak of infection,
DSG2 $2^{\triangle I E C}$ animals displayed higher fecal C. rodentium colonyforming units (CFU) counts, however, both genotypes successfully cleared the infection around day 18 (Suppl. Fig. 11B). Histological analysis demonstrated characteristic epithelial detachment, crypt elongation, and hyperproliferation that were more pronounced in DSG2 ${ }^{\Delta I E C}$ mice (Fig. 5a, b). The latter was confirmed by increased amounts of Ki-67-positive epithelial cells and higher PCNA expression (Fig. 5b, d, Suppl. Fig. 11C). No significant alterations in the amount of goblet cells were seen (Suppl. Fig. 11D). Among the $C$. rodentium-treated animals, $\mathrm{DSG}^{\mathrm{\triangle IEC}}$ mice displayed a 


\section{4}
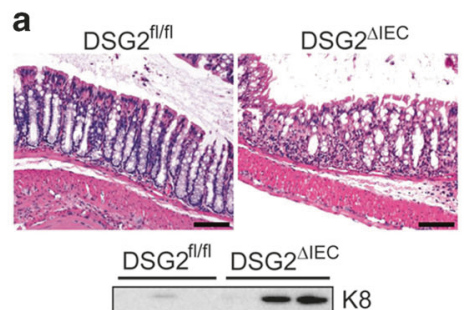

Colon luminal content

C
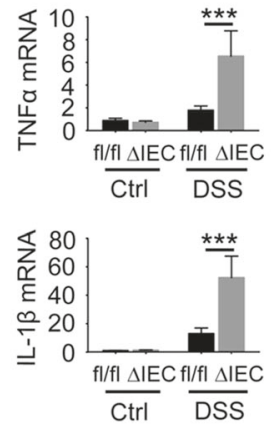

e
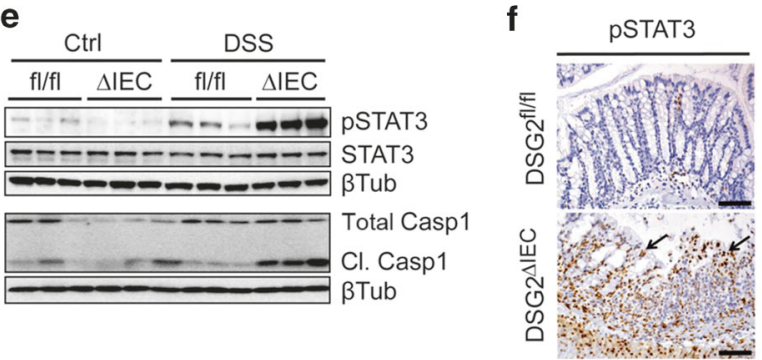

Fig. 4 DSG2-deficient animals (DSG2 ${ }^{\triangle \mathrm{IEC}}$ ) subjected to dextran sodium sulfate (DSS) for 4 days display higher intestinal permeability and enhanced STAT3 signaling. a Hematoxylin and eosin (H\&E) staining of colon sections from DSG2 ${ }^{\triangle \mathrm{IEC}}(\triangle \mathrm{IEC})$ mice and their floxed littermates (DSG2 $2^{\mathrm{f} / \mathrm{fl}}$ or $\mathrm{fl} / \mathrm{fl}$ ) was performed. Western Blot depicts the epithelial cell marker keratin 8 (K8) in the colonic luminal content. b Serum FITC levels were measured following gavage of 4kD FITC-dextran and presented as dot plots $(n=6)$. c-e RT-PCR for TNF $\alpha$, IL-1 $\beta$ and IL-22 as well as the antimicrobial peptide REGIII $\beta$ was performed $(n=4-9)$. Activation of selected signaling pathways was assessed by immunoblotting. The low cytokine expression in nontreated animals (ctrl) was arbitrarily set as 1 . All other values are presented as ratio. L7 (mouse ribosomal protein) gene and $\beta$-tubulin $(\beta T u b)$ were used as an internal and a loading control, respectively. f Immunohistochemistry visualizes phosphorylated STAT3 in colon sections of DSS-treated mice. Scale bar $\mathbf{a}, \mathbf{f}=100 \mu \mathrm{m}$. Two-tailed Student's $t$ test was used for statistical analysis of DSS-treated animals. ${ }^{*} p<0.05,{ }^{* *} p<0.01,{ }^{* * *} p<0.001$

stronger inflammatory reaction, higher expression of proinflammatory cytokines and a higher epithelial cell stress response with activation of the STAT3 signaling pathway and its antimicrobial product Reglll $\beta$ (Fig. 5c, d). Immunostaining revealed that STAT3 was phosphorylated to a large extent in epithelial cells (Fig. $5 d$ ). In contrast to previous in vitro studies, ${ }^{18,19}$ untreated DSG2 ${ }^{\Delta \mathrm{IEC}}$ mice displayed no obvious alteration in EGFR and p38 pathways (not shown). On the other hand, exposure of DSG2 ${ }^{\Delta I E C}$ mice to DSS or C. rodentium resulted in diminished EGFR and p38 levels (Suppl. Fig. 12A,B).

To better understand the biological significance of desmosomal cadherins, we also assessed the role of Dsc2. Similarly to DSG2 ${ }^{\Delta \mathrm{IEC}}$ mice, DSC2 ${ }^{\triangle \mathrm{IEC}}$ mice developed normally (Suppl. Figs. 13). Unlike in DSG2-deficient mice, no profound changes in other desmosomal or TJ components were observed (Fig. 6a, b, Suppl. Figs. 14) and PG immunofluorescence revealed an unaltered desmosomal distribution (Fig. 6b). In addition, no upregulation of other desmocollin and desmoglein isoforms was noticed (Suppl. Fig. 15). Electron microscopy indicated a normal desmosomal ultrastructure (Fig. $6 \mathrm{c}$ ). In line with that, DSC2 ${ }^{\triangle \mathrm{IEC}}$ mice showed no alteration in intestinal permeability (Fig. 6d). A morphologically inconspicuous small and large intestine with properly localized cell types was noted (Fig. 7a, Suppl. Fig. 16). In particular, the amount of goblet cells did not differ between $D S C 2^{\triangle I E C}$ and $\mathrm{DSC}^{\mathrm{f} / \mathrm{fl}}$ mice (Suppl. Fig. 16). Moreover, in both genotypes, treatment with $2.4 \%$ DSS resulted in a comparable weight loss and a similar histological injury (Fig. 7a, b). In line with that, comparable levels of pro-inflammatory cytokines and antimicrobial peptides were noted (Fig. 7c and data not shown). To identify the mechanisms responsible for the different biological impact of DSG2 vs. DSC2 loss, we collected plasma membrane protein fractions. These identified reduced amounts of the cytoprotective chaperone Hsp70 in DSG2 ${ }^{\triangle \mathrm{IEC}}$ but not DSC2 ${ }^{\triangle \mathrm{IEC}}$ animals, whereas the established Dsg2-interacting protein Galectin $3^{20}$ was enriched in the plasma membrane fractions of Dsc2-deficient, but not Dsg2deficient animals (Fig. 7d). As a potential molecular explanation, Hsp70 co-immunoprecipitated with Dsg2 but not with Dsc2 (Fig. 7e and data not shown).

To further understand the ability of Dsg2/Dsc2 to compensate for the loss of its counterpart, we examined colorectal adenocarcinoma DLD1 cells lacking either one or both desmosomal cadherins. ${ }^{18,21}$ Immunofluorescence and immunoblotting confirmed the knockout of both cadherins as well as a complete reexpression of full-length Dsc2 (FL Dsc2) in the double-knockout line (Suppl. Fig. 17A, C). A simultaneous knockout of both cadherins resulted in a marked loss of membranous Dsp/PG staining, that was maintained in the lines lacking only one cadherin (Suppl. Fig. 17B). Immunoblotting demonstrated that the altered Dsp staining pattern is not due to changes in the protein levels (Suppl. Fig. 17C). With regard to functional properties, a knockdown of both cadherins resulted in a stronger decrease in the transepithelial electrical resistance (TER) than the loss of Dsc2 only, while a re-expression of Dsc2 was not sufficient to rescue the TER phenotype of double-knockout cells (Suppl. Fig. 17D).

In summary, our findings uncover the differential biological relevance of the desmosomal cadherins Dsg2 and Dsc2 that are both reduced in the human intestinal injury (Suppl. Fig. 1B, Fig. 7f).

\section{DISCUSSION}

Our study analyzed the in vivo biological role of desmosomal cadherins in the intestine. We showed that desmosomal alterations as well as loss of Dsg2/Dsc2 occur in patients with Crohn's disease. As these patients display a strong activation of TNFa signaling, ${ }^{22}$ our data are in line with previous findings implicating TNFa in the loss of Dsg2 signal. ${ }^{16}$ In that respect, the regulation of Dsg2 is reminiscent of the regulation of TJ proteins, which undergo a strong inflammation-induced remodeling. ${ }^{1,3,4}$ On the other hand, E-cadherin as a key component of AJs remained unaltered in all conditions.

With regard to the biological importance of the desmosomal cadherins, DSG2 ${ }^{\triangle \mathrm{IEC}}$ and DSC2 ${ }^{\triangle \mathrm{IEC}}$ mice displayed morphologically normal desmosomes. In case of DSG2 ${ }^{\triangle I E C}$ animals, this is not surprising since previous reports demonstrated that Dsg2 is not essential for desmosomal assembly. ${ }^{23,24}$ On the other hand, the previous data on Dsc2 were somewhat contradictory. It was reported to be indispensable for desmosomal assembly in an in vitro study, ${ }^{24}$ however, Dsc2 knockdown in cancer cell lines did not result in altered Dsg2 levels. ${ }^{14}$ In addition to normal appearing desmosomes, neither DSG2 $2^{\triangle I E C}$ nor $D S C 2^{\triangle I E C}$ animals show an obvious epithelial injury under basal conditions. This is in line with the intestinal Dsp KOs, that do not exhibit an obvious pathology 
a

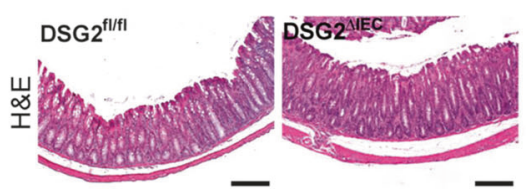

C

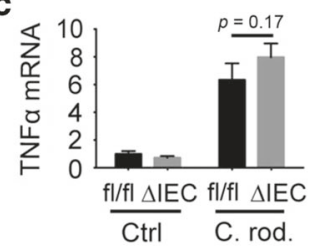

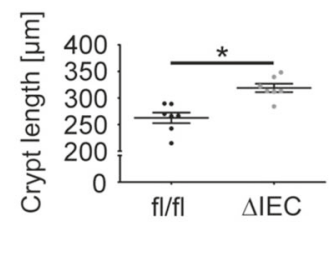

b
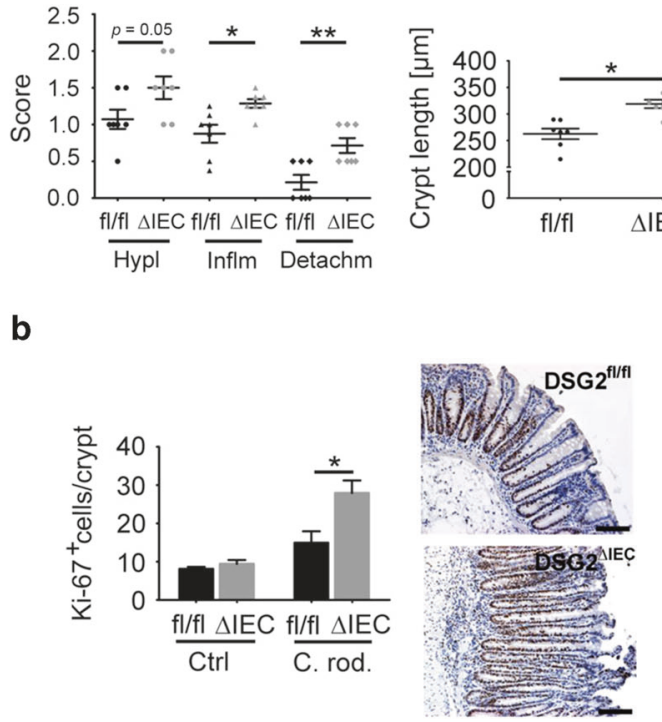
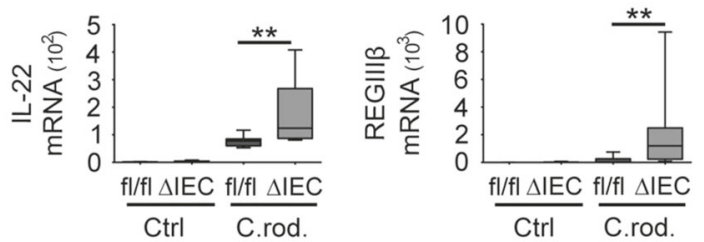

d

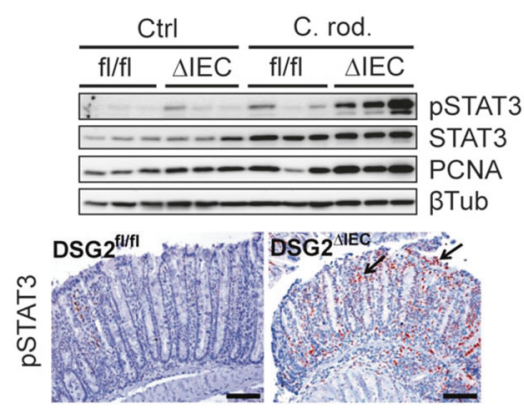

Fig. 5 DSG2-deficient (DSG2 ${ }^{\triangle I E C}$ ) mice exhibit enhanced susceptibility to Citrobacter rodentium (C. rod.)-induced colitis. a Hematoxylin and eosin (H\&E) staining of colon sections with quantification of epithelial hyperplasia (Hypl), detachment (Detachm) and inflammatory immune cell infiltration (Inflm) as well as colon crypt length determination was performed in DSG2 ${ }^{\Delta \mathrm{IEC}}$ mice and their floxed littermates (DSG2 ${ }^{\text {fl/fl }}$ or fl/ fl) 14 days after oral infection with $C$. rod. $(n=7)$. Scale bar $=200 \mu \mathrm{m}$. Data are shown as dot plots. b Ki-67 immunohistochemistry with morphometric quantification visualize the proliferation in colon sections 14 days post infection with C. rod. $(n=7)$. c, d Real time RT-PCR for the highlighted cytokines and the antimicrobial product REGIII $\beta$ as well as immunoblotting for the indicated signaling molecules were conducted in untreated and in C. rod.-infected mice $(n=4-7)$. L7 (mouse ribosomal protein) gene and $\beta$-tubulin ( $\beta$ Tub) were used as an internal and a loading control, respectively. mRNA expression in untreated fl/fl mice was arbitrarily set as 1 and other levels are presented as ratio. Immunohistochemistry illustrates phosphorylated STAT3 in colon sections of $C$. rod.- infected mice. Scale bar $\mathbf{b}, \mathbf{d}=100 \mu \mathrm{m}$. Two-tailed Student's $t$ test (a, b upper panels in $\mathbf{c}$ ) or Mann-Whitney test (lower panels in $\mathbf{c}$ ) were used for statistical analyses of $C . r o d .-e x p o s e d$ mice. ${ }^{*} p<$ $0.05,{ }^{* *} p<0.01$

either. $^{25}$ In contrast, cardiomyocyte-specific DSG2 ablation resulted in spontaneous arrhythmogenic cardiomyopathy, ${ }^{23}$ whereas deletion/mutation of other desmosomal cadherins led to defects in oral epithelia, skin and hair follicles. ${ }^{26}$ Collectively, these data suggest that desmosomal cadherins are essential for integrity of mechanically challenged tissues, but are more dispensable in single-layered epithelia.

Although untreated mice displayed no obvious phenotype, altered desmosomal and TJ protein composition, wider desmosomal space and increased intercellular permeability were noted in $D S G 2^{\triangle I E C}$ but not DSC2 ${ }^{\triangle I E C}$ mice (Fig. 7f). With regard to the desmosomal composition and permeability, our data are consistent with Dsg2/Dsc2 knockdowns performed in cancer cell lines and suggest that Dsg2 loss has a more-profound impact on intercellular junctions than Dsc2 ablation. ${ }^{18,19,23}$ A potential explanation is that homophilic Dsg2 bonds, but not Dsc2 bonds are able to at least partially compensate the ablation of the partner protein. In support of the latter, cell lines that lacked both Dsg2 and Dsc2 displayed similar TER as cells lacking Dsg2 only (Suppl. Fig. 17D). Notably, although homophilic interactions of cadherins occur in epithelial cells, formation of heterophilic DsgDsc interactions is preferred. ${ }^{10,27}$ In addition to the strength of homophilic complexes, interactions with associated proteins might be also responsible for the differences between DSG2 ${ }^{\triangle I E C}$ and $D S C 2^{\triangle I E C}$ mice. In particular, Hsp70 was found to interact with Dsg2 but not Dsc2 and accordingly, Dsg2 ${ }^{\Delta \mathrm{IEC}}$ mice displayed lower Hsp70 levels in their plasma membrane protein fractions. This finding is intriguing as $\mathrm{Hsp70}$ is an established stress-protective protein that ameliorates the development of intestinal injury ${ }^{28,29}$ and is known to interact with keratins, i.e., structures that are functionally tightly linked to desmosomes. ${ }^{30,31}$ However, further studies are needed to delineate the functional importance of the diminished Hsp70 levels in Dsg2 ${ }^{\Delta \mathrm{IEC}}$ mice.

On the other hand, DSC2 $2^{\triangle I E C}$ mice (but not DSG2 $2^{\triangle I E C}$ mice) displayed an accumulation of galectin 3 in their plasma membrane fractions. Of note, galectin 3 constitutes an established Dsg2-binding protein and an important mediator of adhesive strength. ${ }^{20}$ Therefore, a perturbation in Hsp70 might account for the phenotype seen in DSG2 ${ }^{\triangle \mathrm{IEC}}$ mice, whereas galectin 3 may functionally compensate for the Dsc2 loss (Fig. 7f). The reduced p38 levels seen in DSG2 ${ }^{\mathrm{IIEC}}$ mice exposed to DSS or $C$. rodentium might be also of importance, since p38 in intestinal epithelia protects from colitis development. ${ }^{32}$

Although the moderate impairment of intestinal barrier seen in untreated DSG2 ${ }^{\triangle \mathrm{IEC}}$ mice is not sufficient to induce epithelial injury, it becomes more evident in the analyzed stress models. Two key events likely contribute to this finding: (i) inflammatory cytokines, in particularly TNFa, that are induced in the stress models, ${ }^{33,34}$ further weaken the desmosomal adhesion, ${ }^{16}$ (ii) the injuries increase the leakiness of TJs that are known to constitute the major component of the intestinal barrier. The weakening of TJs is both a direct effect of an exposure to DSS/C. rodentium as well as a consequence of the resulting inflammatory reaction. ${ }^{1,3,4,35-37}$ Moreover, our findings indicate that Dsg2 ablation promotes TJ leakiness by decreasing the 

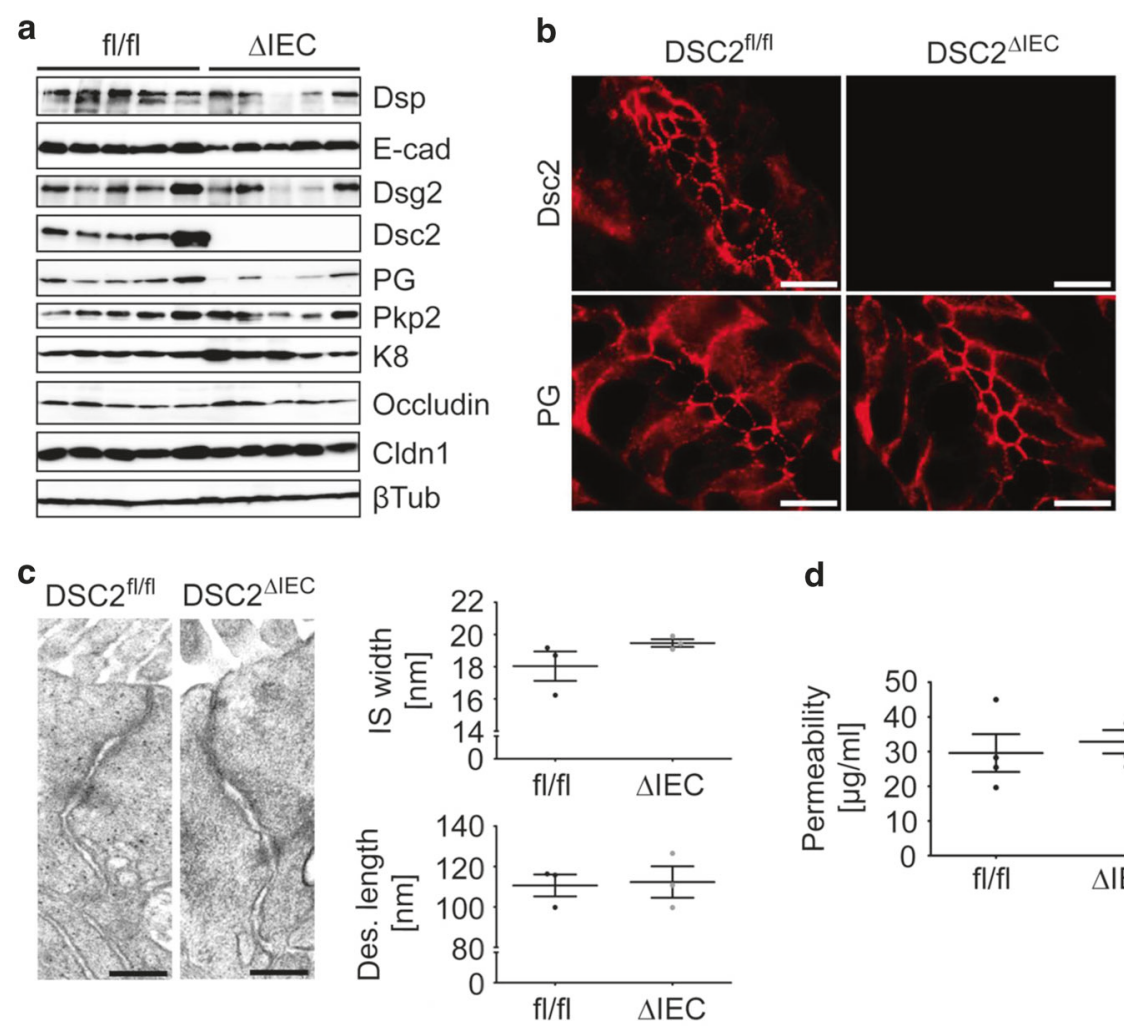

d

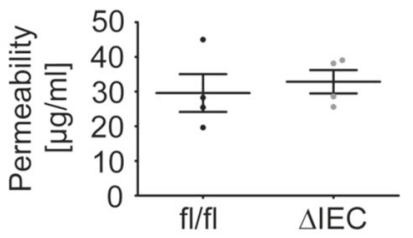

Fig. 6 DSC2-deficient animals (DSC2 $2^{\triangle \mathrm{IEC}}$ ) show an unaltered desmosomal plaque and no increased intestinal permeability. a Immunoblotting was performed on total colon lysates from DSC2 ${ }^{\Delta \mathrm{IEC}}(\Delta \mathrm{IEC})$ mice and their floxed littermates (DSC2 $\left.2^{\mathrm{f} / \mathrm{fl}}, \mathrm{fl} / \mathrm{fl}\right)(n=5)$. $\beta$-tubulin $(\beta T$ Tub) was used as a loading control. $\mathbf{b}$ Immunofluorescence depicts the distribution of Dsc2 and plakoglobin (PG) in the colons of both genotypes. Scale bar = $20 \mu \mathrm{m}$. c Desmosomal ultrastructure was assessed on colon samples by electron microscopy with subsequent quantification of the desmosomal intercellular space width and desmosomal length $(n=3)$. Scale bar $=200 \mathrm{~nm}$. IS = intercellular space. d $4 \mathrm{kD}$ FITC-dextran was administered and serum FITC levels were quantified as a measurement of intestinal permeability $(n=4)$. Data in $\mathbf{c}, \mathbf{d}$ are shown as dot plots. Cldn1, claudin 1; Dsp, desmoplakin; E-cad, E-cadherin; K8, keratin 8; PG, plakoglobin; Pkp2, plakophilin 2

levels of tightening TJ components. This is supported by previous in vitro studies in which loss of Dsg2-mediated adhesion led to disruption of TJ integrity. ${ }^{15,38}$ Consequently, peptides strengthening desmosomal adhesion may present a viable therapeutic strategy in situations with impaired desmosomes. ${ }^{16}$

The above-described loss of epithelial barrier resulted in stronger bacterial translocation that induced the observed intestinal inflammation (Fig. 7f). Two classic inflammatory pathways have been prominently activated: (i) production of pro-inflammatory cytokines IL-1 $\beta$ and TNFa; (ii) activation of IL22 -pSTAT3 signaling. IL-1 $\beta /$ TNFa are known to be produced as a direct reaction to microbial components. ${ }^{39,40}$ The increased levels of the former likely contribute to the stronger epithelial loss seen after DSS treatment thereby leading to a vicious cycle of inflammation and epithelial injury (Fig. 7f). ${ }^{40,41}$ On the other hand, IL-22 belongs to anti-inflammatory cytokines that protect from development of colitis in several models. ${ }^{40,42,43}$ It stimulates, via STAT3 activation, epithelial cell proliferation, and regeneration and likely contributes to the hyperplasia that was observed in the $C$. rodentium model. As both signaling pathways become activated as a consequence of increased epithelial permeability after Dsg2 loss, the balance between IL$1 \beta /$ TNFa and IL-22-pSTAT3 activation presumably dictates, whether DSG2 ${ }^{\triangle \mathrm{IEC}}$ animals will display increased epithelial loss (as seen in the DSS model) or increased regeneration (Citrobacter model).

In summary, our findings reveal desmosomal alterations in patients with Crohn's disease and demonstrate the differential importance of the desmosomal cadherins Dsg2/Dsc2 for the desmosomal structure and susceptibility to intestinal injury (Fig. 7f). Given that a TJ-stabilizing agent showed promise in a clinical trial of celiac disease, ${ }^{44}$ further studies are warranted to explore the therapeutic potential of desmosome-stabilizing peptides. ${ }^{16}$

\section{MATERIALS AND METHODS}

Mouse experiments

Mice with conditional (intestine-specific) Desmoglein 2 (Dsg2) or Desmocollin 2 deletion were generated by crossing previously described DSG2 exon4/5 floxed (DSG2 ${ }^{\mathrm{f} / \mathrm{fl}}$ ) and DSC2 exon2 floxed $\left(\mathrm{DSC2}{ }^{\mathrm{f} / \mathrm{fl}}\right)$ mice with mice expressing Cre under the control of the villin promotor (DSG2 $\left.{ }^{\Delta \mathrm{ECC}} / \mathrm{DSC} 2^{\mathrm{AIE}}\right) .^{23,45-47}$ All mice were on C57BL/6 background and kept under standardized conditions ( $12 \mathrm{~h}$ day/night cycle, $21-24^{\circ} \mathrm{C}$, humidity $\sim 50 \%$ ) with free access to food and water. To induce colitis, 10-week-old sex-matched mice were exposed to dextran sodium sulfate (DSS, MP Biochemicals, Heidelberg, Germany) in the drinking water and killed after 4 days (short-term DSS). Alternatively, DSS was administered for 5 days with a change to normal water afterwards and killing of animals at day 7 (long-term DSS). For DSG2 ${ }^{\triangle \mathrm{IEC}}$ and $\mathrm{DSC}^{\triangle \mathrm{IEC}}$ mice, we used $1.6 \%$ and $2.4 \%$ DSS, respectively. For an infectious model, C. rodentium strain DBS100 ${ }^{48}$ was grown in Luria-Bertani medium at $37^{\circ} \mathrm{C}$ overnight by shaking (200 rpm). 9-10 weeks old sex-matched mice were infected by oral gavage with $1 \times 10^{9} \mathrm{C}$. rodentium and analyzed 14 or 21 days thereafter. At indicated time points, stool was collected, homogenized in sterile phosphate buffered saline (PBS) (Digital Disruptor Genie, Scientific industries, New York, US) and plated in serial dilutions on 
a

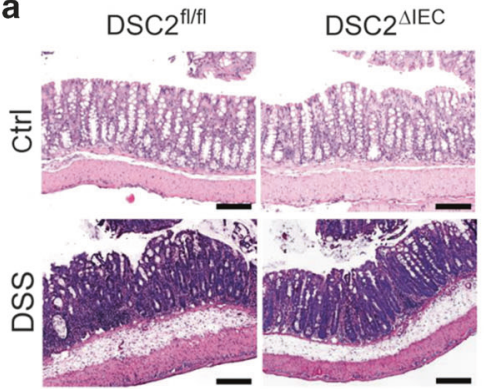

b

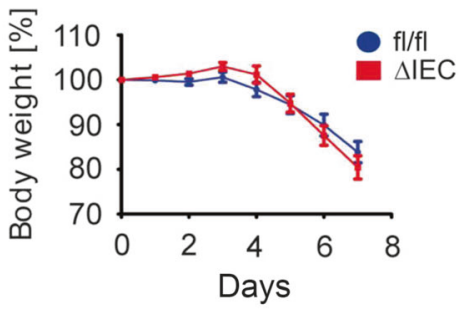

C
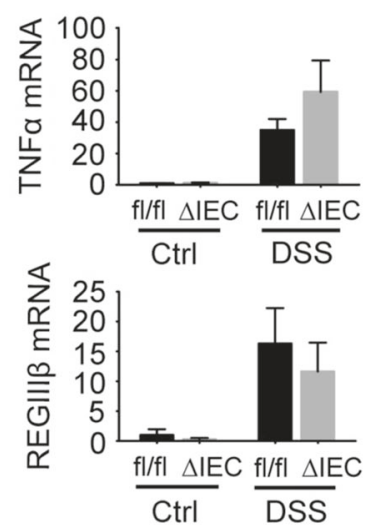

d

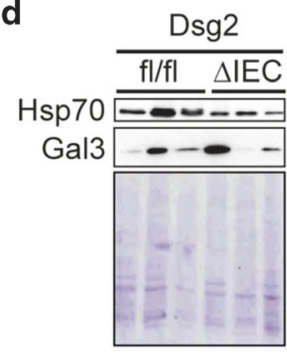

e

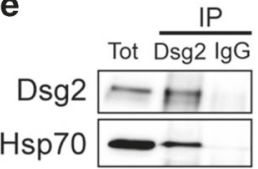

f
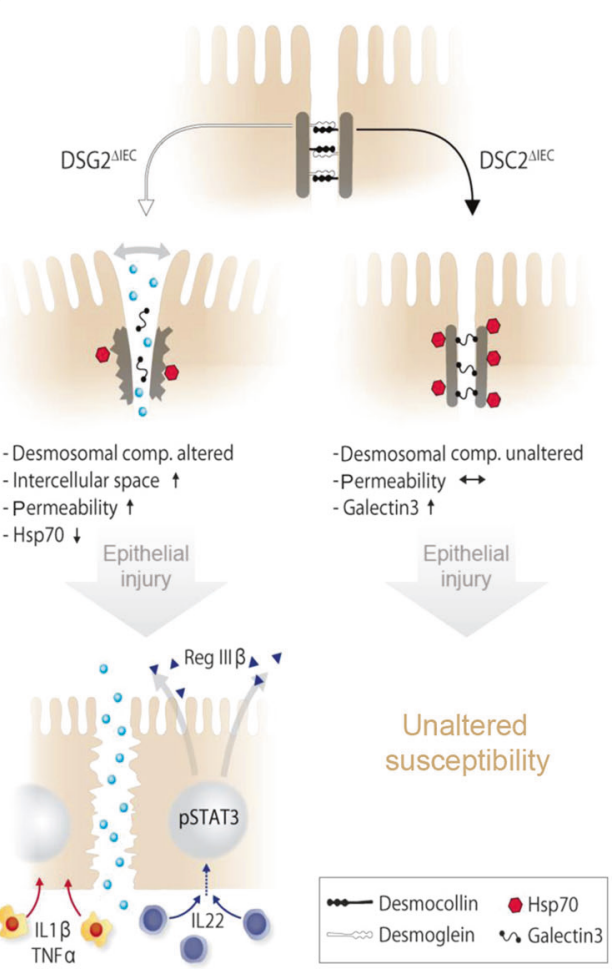

Fig. 7 DSG2 ${ }^{\triangle I E C}$ and DSC2 ${ }^{\triangle I E C}$ mice display differential phenotypes as well as alterations in their plasma membrane proteome. a Overall colon architecture of DSC2 ${ }^{\triangle \mathrm{IEC}}$ mice and corresponding floxed mice (DSC2 ${ }^{\mathrm{fl} / \mathrm{fl}}$ ) was evaluated prior to (ctrl) and 7 days after DSS exposure by hematoxylin and eosin (H\&E) staining. Scale bar $=200 \mu \mathrm{m}$. b The relative body weight of DSC2 ${ }^{\Delta I E C}$ mice (red rectangles, $\left.\Delta I E C\right)$ and their floxed littermates (blue circles, $\mathrm{fl} / \mathrm{fl}$ ) was measured daily starting at the day of the first DSS administration $(n=7)$. c The mRNA levels of the proinflammatory cytokine TNF $\alpha$ and the antimicrobial peptide REGIII $\beta$ were evaluated prior to (ctrl) and 7 days after DSS exposure by real time RTPCR. The cytokine expression in ctrl animals was arbitrarily set as 1 . L7 (mouse ribosomal protein) gene was used as an internal control. d Plasma membrane protein fractions were isolated from colons of both genotypes and subjected to immunoblotting. Coomassie blue staining of blots was used as loading control. Gal3, Galectin 3; Hsp70, heat shock protein 70. e Colonic mucosa lysates from fl/fl animals were used for co-immunoprecipitation with Dsg2 (left panel) and Dsc2 (right panel), that was followed by immunoblotting. IgG-conjugated beads (IgG) and total lysates (Total) were used as a control and an input, respectively. $f$ Schematic illustrates the molecular changes seen in DSG2 ${ }^{\Delta I E C}$ and DSC2 ${ }^{\mathrm{IIEC}}$ mice

MacConkey agar plates (Roth, Karlsruhe, Germany) to count CFU of C. rodentium. Untreated, age- and sex-matched littermates were used as controls.

To examine intestinal permeability, mice were fasted for $3 \mathrm{~h}$ and gavaged with $0.6 \mathrm{mg} / \mathrm{g}$ of body weight $4 \mathrm{kD}$ fluorescein isothiocyanate-labeled dextran (Sigma-Aldrich, Steinheim, Germany). After $4 \mathrm{~h}$, blood was collected retroorbitally and the fluorescence intensity in serum was measured (excitation: $492 \mathrm{~nm}$; emission: $525 \mathrm{~nm}$, Cytation3 imaging reader, BioTek, Bad Friedrichshall, Germany). The samples were prepared in duplicates and the results calculated according to the standard curve. All animals were weighted and killed by an isoflurane overdose with a subsequent cervical dislocation. In selected animals, the distal colon was analyzed via a mini-endoscope (Karl Storz, Tuttlingen, Germany). Rectal bleeding was examined using commercial hemoCARE fecal occult blood Guajak test using a semiquantitative scoring from 0 to 3 (0: no bleeding, 1: mild bleeding, 2: moderate bleeding, 3: severe bleeding). Mesenteric lymph nodes were dissected and homogenates were plated on Columbia sheep blood agar plates (Oxoid/Thermo Scientific, Munich, Germany) to evaluate the translocation of bacteria into the lymph nodes. Proximal intestinal parts were washed and stored as Swiss rolls in $4 \%$ formaldehyde for histological evaluation or embedded in O.C.T. compound (Tissue-Tek, Sakura, Staufen, Germany) for cryosectioning. Distal parts were washed and snap frozen in liquid nitrogen for biochemical and RNA analysis.
Human samples

As described in detail previously, ${ }^{38}$ human specimens were obtained from terminal ileum of patients who suffered from refractory $C D$ and/or had a complication that required surgical resection, such as stenosis, fistula, or abscesses. Control tissue samples of the terminal ileum were from patients that required right hemicolectomy in which the surgical resection routinely involves a part of the healthy small intestine (for further information see Suppl. Table 1). For western blot analyses, mucosa was mechanically dissected from the tissue immediately after the resection and transferred into lysis buffer containing $25 \mathrm{mmol} / \mathrm{L} 4-$ (2-hydroxyethyl)-1-piperazineethanesulfonic acid, $2 \mathrm{mmol} / \mathrm{L}$ ethylenediaminetetraacetic acid (EDTA), $25 \mathrm{mmol} / \mathrm{L} \mathrm{NaF}$, and $1 \%$ SDS. Specimens were homogenized with TissueLyzer (Qiagen, Hilden, Germany) and normalized with BCA assay (Thermo Fisher, Waltham, MA). Anti-Dsg2 (Invitrogen, Carlsbad, CA), anti-Dsc2 (Abcam, Cambridge, UK) and anti-E-cadherin (BD Biosciences, Franklin Lakes, NJ) primary antibodies were combined with a horseradish peroxidase-labeled goat anti-mouse antibody (Dianova, Hamburg, Germany).

A second part of the tissue samples was fixed in $4 \%$ paraformaldehyde, embedded in paraffin and cut into 1- $\mu \mathrm{m}$ thick sections. Immunostaining was performed as described previously $^{49}$ using rabbit anti-Dsg2 (Invitrogen), anti-Dsc2 (Abcam), and mouse anti-E-cadherin (BD Biosciences) antibodies in combination with a Cy3-labeled goat anti-mouse- and a Cy2- 
labeled goat anti-rabbit antibody (Both Dianova, Hamburg, Germany). To analyze the tissue architecture, all specimens were stained with Hematoxylin and Eosin (H\&E).

Cell culture experiments

For the described experiments, we used previously published DLD1 cells with CRISPR/Cas9-mediated knockdown of Dsc2 $(\triangle \mathrm{Dsc} 2)$ or both desmosomal cadherins $(\Delta \mathrm{Dsg} 2 \Delta \mathrm{Dsc} 2)$, as well as a cell line in that the knockout of both cadherins was followed by a re-expression of Dsc2 $(\Delta \mathrm{Dsg} 2 \Delta \mathrm{Dsc} 2+\mathrm{FL} \mathrm{Dsc2}){ }^{21}$ The cells were cultured in Dulbecco's modified Eagle's medium (Life Technologies, Carlsbad, CA) containing 10\% fetal bovine serum (Biochrom, Berlin, Germany), $50 \mathrm{U} / \mathrm{ml}$ penicillin and $50 \mathrm{U} / \mathrm{ml}$ streptomycin (AppliChem, Darmstadt, Germany) in $5 \% \mathrm{CO}_{2}$ atmosphere at $37^{\circ} \mathrm{C}$ until they reached confluence. ${ }^{18}$

\section{Biochemical methods}

Total tissue lysates were prepared by homogenization of tissues in $3 \%$ sodium dodecyl sulphate (SDS)-containing buffer with protease and phosphatase inhibitors. To determine luminal protein composition, colon was removed, opened longitudinally, and vigorously inverted 15 times in PBS. The solution containing epithelial cells was centrifuged at $5000 \mathrm{rpm}$ for $10 \mathrm{~min}$ at $4{ }^{\circ} \mathrm{C}$ and the pellet was used for homogenization. DLD1 cells were grown in 24-well plates and lysed using 1\% SDS lysis buffer supplemented with a protease-inhibitor cocktail (Roche, Mannheim, Germany). Same amounts of proteins were separated by SDS-polyacrylamide gel electrophoresis (SDS-PAGE) followed by transfer to PVDF/ nitrocellulose membranes or staining with $0.1 \%$ Coomassie Brilliant Blue G-250. The membranes were incubated with specific primary and horseradish peroxidase-coupled secondary antibodies, which were then visualized by an enhanced chemiluminescence detection kit (GE Healthcare/Amersham Biosciences, UK). The antibodies used in this study are summarized in Suppl. Table 3.

To determine myeloperoxidase activity, colonic tissue was homogenized in ice-cold $50 \mathrm{mM}$ potassium phosphate buffer containing $0.5 \%$ hexadecyltrimethylammonium bromide. Equal volume of cell lysis buffer was added and the homogenates were freeze-thawed twice. After centrifugation, supernatant was removed and supplemented with reaction buffer (with $O_{-}$ dianisidine hydrochloride and $0.001 \% \mathrm{H}_{2} \mathrm{O}_{2}$ ) or standard solution. After $3 \mathrm{~min}$ of reaction time, the absorbance was spectrophotometrically measured at $450 \mathrm{~nm}$ (Cytation3 imaging reader). The samples were prepared in duplicates and the results were calculated according to the standards.

Plasma membrane protein fractions from colon scrapings were extracted according to the manufacturer's protocol (Abcam, ab65400). In brief, scrapings were homogenized in an appropriate volume of homogenization buffer containing protease-inhibitor cocktail. Following centrifugation, the pellet that contains plasma membrane proteins was re-suspended in the provided upper phase solution. Plasma membrane proteins were purified by repeated addition of lower phase solution that was later on removed by centrifugation at low speed. Finally, the plasma membrane proteins were pelleted via high-speed centrifugation at $+4{ }^{\circ} \mathrm{C}$. The membrane fraction was dissolved in $0.5 \%$ Triton- $\mathrm{X}$ 100/PBS that was supplemented with Laemmli buffer (0.2 M Tris $\mathrm{pH} 6.8,40 \%$ glycerol, $8 \%$ SDS, $7.2 \%$ $\beta$-mercaptoethanol, $0.02 \%$ bromophenol blue).

\section{Immunoprecipitation}

Total lysates were prepared by homogenization of colon scrapings in radioimmunoprecipitation assay buffer $(2.5 \mathrm{mM}$ Tris- $\mathrm{HCl} \mathrm{pH} \mathrm{7.4,}$ $0.025 \%$ sodium deoxycholate, $150 \mathrm{mM} \mathrm{NaCl}, 2 \mathrm{mM}$ EDTA, $0.01 \%$ NP-40). After conjugation of Dsc2 or Dsg2 antibody to Protein G Dynabeads (Thermo Fisher) for $10 \mathrm{~min}$, the lysates were added to the beads-antibody complex and the solution was incubated upon a gentle rotation for $2 \mathrm{~h}$ at $+4{ }^{\circ} \mathrm{C}$. A magnet was used to collect the beads that were washed three times with a supplied washing buffer. The supernatant was discarded, the proteins were eluted via heating in SDS-containing Laemmli buffer for $5 \mathrm{~min}$ at $95^{\circ} \mathrm{C}$ and further analyzed by SDS-PAGE.

\section{Histological analysis}

The formaldehyde-fixed tissues were paraffin-embedded, cut into $3 \mu \mathrm{m}$-thick sections and stained with H\&E or periodic acid-Schiff (PAS). For the latter, deparaffinized slides were oxidized in $2 \%$ periodic acid solution for $5 \mathrm{~min}$. After washing in distilled water, a staining with Schiff reagent for $15 \mathrm{~min}$ was performed and followed by hematoxylin counterstaining. Finally, the sections were blued in $1 \mathrm{M}$ Tris buffer $(\mathrm{pH}$ 8). Images were recorded with a Zeiss light microscope (Zeiss, Germany) and AxioVision Rel 4.8 software (Zeiss, Germany). PAS-positive cells were counted as a mean from at least thirty different crypts by ImageJ software.

A previously described, semi-quantitative histopathological score $^{50}$ with minor modifications was used for evaluation of DSS-treated samples. The following parameters were assessed: (i) submucosa thickening/edema, (ii) inflammatory cell infiltration, (iii) goblet cell loss (each parameter with a score 0-3: 0: normal; 1: mild; 2: moderate; 3: severe), (iv) epithelial damage/erosion (0: normal; $2:<1 / 3$ of total area with altered epithelial cell morphology; $4:>1 / 3$ of total area with altered epithelial cell morphology and/or mild erosions; $6:<10 \%$ of ulcerative areas; 8 : $10-20 \%$ of ulcerative areas, $10:>20 \%$ of ulcerative areas).

The $C$. rodentium exposed colons were semi-quantitatively scored. Following criteria were assessed: (i) epithelial detachment (0: normal, 0,5: low, 1: high); (ii) hyperplasia (0: normal, 1: low hyperplasia, 2: high hyperplasia); (iii) inflammation (0: normal, 1: moderate, 2: severe). Lengths of colonic crypts were measured in longitudinal orientation. At least 10 crypts per mouse were analyzed and the results are presented as means. All analyses were performed in a blinded manner by $A G$ and an experienced pathologist (PB).

\section{Study approval}

The animal experiments were approved by the state of North Rhine-Westphalia in Germany and the University of Aachen animal care committee and were conducted in compliance with the German Law for Welfare of Laboratory Animals. All CD patients had given their informed consent before surgery, and the study was approved by the Ethical Board of the University of Würzburg (proposal numbers 113/13 and 46/11).

Data analysis and statistical methods

Image quantifications were performed with ImageJ (National Institutes of Health, Bethesda, USA). Data were analyzed with an unpaired two-tailed Student's $t$ test, Mann-Whitney test or oneway analysis of variance where appropriate. $P$ values below 0.05 were considered as statistically significant.

\section{ACKNOWLEDGEMENTS}

We are thankful to Shintaro T. Suzuki for providing the DLD1 cell lines and Adam Breitscheidel for his assistance with figure preparation. The expert technical assistance of Linda Schaub, Ingrid Breuer, Silvia Roubrocks, Ana Mandić, Sandra Jumpertz, Sabine Eisner, and Silvia Koch is gratefully acknowledged. Our work was supported by a grant from the Interdisciplinary Centre for Clinical Research (IZKF) within the faculty of Medicine at the RWTH Aachen University, by Else Kröner Exzellenzstipendium (to P.S.), by the Deutsche Forschungsgemeinschaft (DFG) SFB TRR57 (to PS, PB, and CT), SFB 985 (to PS and CT), and by the German Federal Ministry of Education and Research (BMBF01GM1518A to PB). The research on epithelial junctions is supported by the DFG Priority Program SPP 1782 to NS, PS, RL, and JW. 
Desmoglein 2, but not desmocollin 2, protects intestinal epithelia from...

\section{AUTHOR CONTRIBUTIONS}

Study was planned and designed by A.G., R.E.L. and P.S. and the acquisition of data was performed by A.G., L.A.P.P., G.M.S., M.M., P.B., H.U. and C.P. Analysis and interpretation of data were conducted by A.G., N.S., P.B., G.S., R.E.L., J.W., P.S. and C.P. A.G. and P.S. drafted the manuscript and all authors contributed to the critical revision of the manuscript for important intellectual content. Statistical analysis was performed by A.G. and P.S., who also obtained the funding and supervised the study. S.K., C.A.K., G.S., C.T., R.E.L., N.G. and A.H. provided technical or material support.

\section{ADDITIONAL INFORMATION}

The online version of this article (https://doi.org/10.1038/s41385-018-0062-z) contains supplementary material, which is available to authorized users.

Competing interests: The authors declare no competing interests.

\section{REFERENCES}

1. Turner, J. R. Intestinal mucosal barrier function in health and disease. Nat. Rev. Immunol. 9, 799-809 (2009).

2. Barmeyer, C., Schulzke, J. D. \& Fromm, M. Claudin-related intestinal diseases. Semin. Cell Dev. Biol. 42, 30-38 (2015).

3. Turner, J. R., Buschmann, M. M., Romero-Calvo, I., Sailer, A. \& Shen, L. The role of molecular remodeling in differential regulation of tight junction permeability. Semin. Cell Dev. Biol. 36, 204-212 (2014).

4. Capaldo, C. T. \& Nusrat, A. Claudin switching: physiological plasticity of the tight Junction. Semin. Cell Dev. Biol. 42, 22-29 (2015).

5. Nekrasova, O. \& Green, K. J. Desmosome assembly and dynamics. Trends Cell Biol. 23, 537-546 (2013)

6. Holthofer, B., Windoffer, R., Troyanovsky, S. \& Leube, R. E. Structure and function of desmosomes. Int. Rev. Cytol. 264, 65-163 (2007).

7. Wang, H. et al. Desmoglein 2 is a receptor for adenovirus serotypes $3,7,11$ and 14. Nat. Med. 17, 96-104 (2011).

8. Brooke, M. A., Nitoiu, D. \& Kelsell, D. P. Cell-cell connectivity: desmosomes and disease. J. Pathol. 226, 158-171 (2012).

9. De Arcangelis, A. et al. Hemidesmosome integrity protects the colon against colitis and colorectal cancer. Gut 66, 1748-1760 (2016).

10. Harrison, O. J. et al. Structural basis of adhesive binding by desmocollins and desmogleins. Proc. Natl Acad. Sci. USA 113, 7160-7165 (2016).

11. Garrod, D. \& Chidgey, M. Desmosome structure, composition and function. Biochim. Biophys. Acta 1778, 572-587 (2008).

12. Delva, E., Tucker, D. K. \& Kowalczyk, A. P. The desmosome. Cold Spring Harb. Perspect. Biol. 1, a002543 (2009).

13. Kowalczyk, A. P. \& Green, K. J. Structure, function, and regulation of desmosomes. Progress. Mol. Biol. Transl. Sci. 116, 95-118 (2013).

14. Kolegraff, K., Nava, P., Helms, M. N., Parkos, C. A. \& Nusrat, A. Loss of desmocollin-2 confers a tumorigenic phenotype to colonic epithelial cells through activation of Akt/beta-catenin signaling. Mol. Biol. Cell 22, 1121-1134 (2011).

15. Nava, P. et al. Desmoglein-2: a novel regulator of apoptosis in the intestinal epithelium. Mol. Biol. Cell 18, 4565-4578 (2007).

16. Spindler, V. et al. Loss of desmoglein 2 contributes to the pathogenesis of Crohn's disease. Inflamm. Bowel Dis. 21, 2349-2359 (2015).

17. Raczynski, A. R. et al. Enteric infection with Citrobacter rodentium induces coagulative liver necrosis and hepatic inflammation prior to peak infection and colonic disease. PLOS. ONE 7, e33099 (2012).

18. Ungewiss, $H$. et al. Desmoglein 2 regulates the intestinal epithelial barrier via p38 mitogen-activated protein kinase. Sci. Rep. 7, 6329 (2017).

19. Kamekura, R. et al. Loss of the desmosomal cadherin desmoglein-2 suppresses colon cancer cell proliferation through EGFR signaling. Oncogene 33, 4531-4536 (2014).

20. Jiang, K. et al. Galectin 3 regulates desmoglein-2 and intestinal epithelial intercellular adhesion. J. Biol. Chem. 289, 10510-10517 (2014)

21. Fujiwara, M. et al. Desmocollin-2 alone forms functional desmosomal plaques, with the plaque formation requiring the juxtamembrane region and plakophilins. J. Biochem. 158, 339-353 (2015).

22. Atreya, R. et al. In vivo imaging using fluorescent antibodies to tumor necrosis factor predicts therapeutic response in Crohn's disease. Nat. Med. 20, 313-318 (2014).

23. Kant, S., Holthofer, B., Magin, T. M., Krusche, C. A. \& Leube, R. E. Desmoglein 2dependent arrhythmogenic cardiomyopathy is caused by a loss of adhesive function. Circ. Cardiovasc. Genet. 8, 553-563 (2015).
24. Lowndes, M. et al. Different roles of cadherins in the assembly and structural integrity of the desmosome complex. J. Cell Sci. 127, 2339-2350 (2014).

25. Sumigray, K. D. \& Lechler, T. Desmoplakin controls microvilli length but not cell adhesion or keratin organization in the intestinal epithelium. Mol. Biol. Cell 23 792-799 (2012).

26. Thomason, H. A., Scothern, A., McHarg, S. \& Garrod, D. R. Desmosomes: adhesive strength and signalling in health and disease. Biochem. J. 429, 419-433 (2010).

27. Nie, Z., Merritt, A., Rouhi-Parkouhi, M., Tabernero, L. \& Garrod, D. Membraneimpermeable cross-linking provides evidence for homophilic, isoform-specific binding of desmosomal cadherins in epithelial cells. J. Biol. Chem. 286, 2143-2154 (2011).

28. Samborski, P. \& Grzymislawski, M. The role of HSP70 heat shock proteins in the pathogenesis and treatment of inflammatory bowel diseases. Adv. Clin. Exp. Med. 24, 525-530 (2015).

29. Tanaka, K. et al. Genetic evidence for a protective role for heat shock factor 1 and heat shock protein 70 against colitis. J. Biol. Chem. 282, 23240-23252 (2007).

30. Osmani, N. \& Labouesse, M. Remodeling of keratin-coupled cell adhesion complexes. Curr. Opin. Cell Biol. 32, 30-38 (2015).

31. Hatzfeld, M., Keil, R. \& Magin, T. M. Desmosomes and intermediate filaments: their consequences for tissue mechanics. Cold Spring Harb. Perspect. Biol. 9, a029157 (2017).

32. Otsuka, M. et al. Distinct effects of p38alpha deletion in myeloid lineage and gut epithelia in mouse models of inflammatory bowel disease. Gastroenterology 138 1255-1265 (2010).

33. Kojouharoff, G. et al. Neutralization of tumour necrosis factor (TNF) but not of IL-1 reduces inflammation in chronic dextran sulphate sodium-induced colitis in mice. Clin. Exp. Immunol. 107, 353-358 (1997).

34. Goncalves, N. S. et al. Critical role for tumor necrosis factor alpha in controlling the number of lumenal pathogenic bacteria and immunopathology in infectious colitis. Infect. Immun. 69, 6651-6659 (2001).

35. Poritz, L. S. et al. Loss of the tight junction protein ZO-1 in dextran sulfate sodium induced colitis. J. Surg. Res. 140, 12-19 (2007).

36. Flynn, A. N. \& Buret, A. G. Tight junctional disruption and apoptosis in an in vitro model of Citrobacter rodentium infection. Microb. Pathog. 45, 98-104 (2008).

37. Nighot, P. et al. Matrix metalloproteinase 9-induced increase in intestinal epithelial tight junction permeability contributes to the severity of experimental DSS colitis. Am. J. Physiol. Gastrointest. Liver Physiol. 309, G988-G997 (2015).

38. Schlegel, N. et al. Desmoglein 2-mediated adhesion is required for intestinal epithelial barrier integrity. Am. J. Physiol. Gastrointest. Liver Physiol. 298, G774-G783 (2010).

39. Kontoyiannis, D., Pasparakis, M., Pizarro, T. T., Cominelli, F. \& Kollias, G. Impaired on/off regulation of TNF biosynthesis in mice lacking TNF AU-rich elements: implications for joint and gut-associated immunopathologies. Immunity 10, 387-398 (1999).

40. Neurath, M. F. Cytokines in inflammatory bowel disease. Nat. Rev. Immunol. 14, 329-342 (2014).

41. Carvalho, F. A. et al. Interleukin-1 beta (IL-1 beta) promotes susceptibility of Tolllike receptor 5 (TLR5) deficient mice to colitis. Gut 61, 373-384 (2012).

42. Sabat, R., Ouyang, W. \& Wolk, K. Therapeutic opportunities of the IL-22-IL-22R1 system. Nat. Rev. Drug. Discov. 13, 21-38 (2014).

43. Pickert, G. et al. STAT3 links IL-22 signaling in intestinal epithelial cells to mucosal wound healing. J. Exp. Med. 206, 1465-1472 (2009).

44. Leffler, D. A. et al. Larazotide acetate for persistent symptoms of celiac disease despite a gluten-free diet: a randomized controlled trial. Gastroenterology 148 1311-1319 e1316 (2015).

45. el Marjou, F. et al. Tissue-specific and inducible Cre-mediated recombination in the gut epithelium. Genesis 39, 186-193 (2004).

46. Madison, B. B. et al. Cis elements of the villin gene control expression in restricted domains of the vertical (crypt) and horizontal (duodenum, cecum) axes of the intestine. J. Biol. Chem. 277, 33275-33283 (2002).

47. Rimpler U. Funktionelle Charakterisierung von Desmocollin 2 während der Embryonalentwicklung und im adulten Herzen in der Maus. Humboldt University zu Berlin (2014).

48. Schauer, D. B. \& Falkow, S. The eae gene of Citrobacter freundii biotype 4280 is necessary for colonization in transmissible murine colonic hyperplasia. Infect. Immun. 61, 4654-4661 (1993).

49. Meir, M. et al. Glial cell line-derived neurotrophic factor promotes barrier maturation and wound healing in intestinal epithelial cells in vitro. Am. J. Physiol. Gastrointest. Liver Physiol. 309, G613-G624 (2015).

50. Chinen, T. et al. Prostaglandin E2 and SOCS1 have a role in intestinal immune tolerance. Nat. Commun. 2, 190 (2011). 